\title{
Simultaneous ground-based and satellite observations of Pc5 geomagnetic pulsations: A case study using multipoint measurements
}

\author{
S.-K. Sung ${ }^{1,2}$, K.-H. Kim ${ }^{3}$, D.-H. Lee ${ }^{2}$, K. Takahashi ${ }^{4}$, C. A. Cattell ${ }^{5}$, M. André ${ }^{6}$, Y. V. Khotyaintsev ${ }^{6}$, and A. Balogh ${ }^{7}$ \\ ${ }^{1}$ Astronomy Program, School of Earth and Environmental Science, Seoul National University, Seoul, Korea \\ ${ }^{2}$ Department of Astronomy and Space Science, Kyung Hee University, Kyunggi, Korea \\ ${ }^{3}$ Korea Astronomy and Space Science Institute, Taejeon, Korea \\ ${ }^{4}$ Applied Physics Laboratory, Johns Hopkins University, Laurel, Maryland, U.S.A. \\ ${ }^{5}$ School of Physics and Astronomy, University of Minnesota, Minneapolis, Minnesota, U.S.A. \\ ${ }^{6}$ Swedish Institute of Space Physics, Uppsala, Sweden \\ ${ }^{7}$ Imperial College, London, U.K.
}

(Received November 4, 2005; Revised March 8, 2006; Accepted March 8, 2006; Online published July 26, 2006)

\begin{abstract}
Pc5 pulsations with a latitude-independent frequency of $\sim 2.8 \mathrm{mHz}$ (period $\sim 6 \mathrm{~min}$ ) were observed with ground-based magnetometers in the morning sector (0700-1000 local time) from 0730 to 0810 UT, April 29, 2001. The pulsations exhibited an amplitude peak and a $\sim 180^{\circ}$ phase change at $\sim 67^{\circ}$ geomagnetic latitude. A vortex structure of the equivalent ionospheric current system was also observed centered at the geomagnetic latitude between $67^{\circ}$ and $71^{\circ}$. During the Pc5 event, the Polar spacecraft was located near the morningside magnetic equator and crossed magnetic field lines conjugate to the ground stations at which the pulsations were strong. Polar observed $\sim 2.8 \mathrm{mHz}$ pulsations in the radial electric field and compressional magnetic field components. Since toroidal mode Alfvén waves in the magnetosphere are characterized by an electric field perturbation in the radial direction, the simultaneous presence of pulsations in both components indicates that a field line resonance (FLR) was driven by compressional pulsations. The ground $H$ component at the station conjugate to Polar and the radial electric field perturbation at Polar oscillated with a great similarity without a phase delay. From an analysis of the ground-satellite data, we suggest that the ground perturbations are associated with toroidal mode Alfvén waves rather than compressional mode propagating across magnetic field lines. In addition, we discuss the source of the compressional perturbations observed at Polar using solar wind and geosynchronous data.
\end{abstract}

Key words: Pc5, field line resonance, simultaneous observation.

\section{Introduction}

Pc5 geomagnetic pulsations (period $\sim 150-600$ s) are commonly observed at high-latitudes and tend to have their maximum amplitudes at a geomagnetic latitude between $\sim 65^{\circ}$ and $\sim 75^{\circ}$ (e.g., Samson and Rostoker, 1972). The latitude of the peak amplitude increases with decreasing frequency (Mathie et al., 1999). The latitude dependence of amplitude enhancement has been interpreted as field line resonance (FLR) (Southwood, 1974; Chen and Hasegawa, 1974) in the background Alfvén continuum of field line eigenfrequencies. An early ground-based study by Samson et al. (1971) reported that the sense of polarization of Pc5 pulsations changes at the latitude where the amplitude is maximum. Such variations in polarization and amplitude signatures with latitude are well explained by FLR due to the coupling of a quasi-monochromatic surface wave produced at the magnetopause by the Kelvin-Helmholtz (K-H) instability to a shear Alfvén wave. The K-H surface waves drive compressional waves into the magnetosphere. The most efficient coupling occurs at locations where the eigen-

Copyright (c) The Society of Geomagnetism and Earth, Planetary and Space Sciences (SGEPSS); The Seismological Society of Japan; The Volcanological Society of Japan; The Geodetic Society of Japan; The Japanese Society for Planetary Sciences; TERRAPUB frequencies of the field lines match the frequencies of the $\mathrm{K}-\mathrm{H}$ waves.

It has been generally accepted that compressional pulsations in the Pc5 band can be generated at the magnetopause by solar wind dynamic pressure variations (e.g., Sibeck et al., 1989; Sibeck, 1994; Korotova and Sibeck, 1994; Kim et al., 2002). Such compressional oscillations propagate radially inward from the magnetopause and can couple to shear Alfvén waves at those locations where the driver frequency matches the local shear Alfvén wave frequency (Lee and Lysak, 1991). This process is called field line resonance (FLR). In such cases, the compressional waves are associated with external pressure variations and mimic the behavior of the K-H surface waves. In addition to a periodic source, an impulsive (broadband) source can generate FLRs. In this case, the frequencies vary with $L$ value or magnetic latitude as shown in several numerical simulations. Therefore, simultaneous observations in space and on the ground and careful inspection of solar wind conditions is required to identify the source mechanism of Pc5 pulsations.

High-latitude ground-based studies have reported stable Pc5 pulsations having discrete frequencies of 1.3, 1.9, 2.6, 3.4 and $4.2 \mathrm{mHz}$ (e.g., Samson et al., 1991, 1992a, b; Ruo- 
Table 1. List of the IMAGE Stations.

\begin{tabular}{|c|c|c|c|c|c|}
\hline \multicolumn{2}{|c|}{ Stations } & \multicolumn{2}{|c|}{ Geographic } & \multicolumn{2}{|c|}{ Corrected Geomagnetic } \\
\hline Name & Code & Latitude & Longitude & Latitude & Longitude \\
\hline Ny Ålesund & NAL & 78.92 & 11.95 & 76.19 & 111.54 \\
\hline Longyearbyen & LYR & 78.20 & 15.82 & 75.24 & 112.36 \\
\hline Hornsund & HOR & 77.00 & 15.60 & 74.13 & 109.85 \\
\hline Hopen Island & HOP & 76.51 & 25.01 & 73.05 & 115.39 \\
\hline Bear Island & $\mathrm{BJN}$ & 74.50 & 19.20 & 71.44 & 108.30 \\
\hline Sørøya & SOR & 70.54 & 22.22 & 67.34 & 106.37 \\
\hline Troms $\emptyset$ & TRO & 69.66 & 18.94 & 66.64 & 103.09 \\
\hline Andenes & AND & 69.30 & 16.03 & 66.45 & 100.54 \\
\hline Kilpisjärvi & KIL & 69.02 & 20.79 & 65.88 & 103.99 \\
\hline Leknes & LEK & 68.13 & 13.54 & 65.41 & 97.65 \\
\hline Abisko & $\mathrm{ABK}$ & 68.35 & 18.82 & 65.30 & 101.92 \\
\hline Ivalo & IVA & 68.56 & 27.29 & 65.09 & 108.77 \\
\hline Muonio & MUO & 68.02 & 23.53 & 64.72 & 105.40 \\
\hline Kiruna & KIR & 67.84 & 20.42 & 64.69 & 102.81 \\
\hline Lovozero & LOZ & 67.97 & 35.08 & 64.21 & 114.70 \\
\hline Sodankylä & SOD & 67.37 & 26.63 & 63.92 & 107.45 \\
\hline Pello & PEL & 66.90 & 24.08 & 63.55 & 105.10 \\
\hline Rørvik & RVK & 64.94 & 10.98 & 62.25 & 93.43 \\
\hline Lycksele & LYC & 64.61 & 18.75 & 61.45 & 99.43 \\
\hline Dombås & DOB & 62.07 & 9.11 & 59.31 & 90.30 \\
\hline
\end{tabular}

honiemi et al., 1991; Mathie et al., 1999; Mathie and Mann, 2000). Such discrete frequencies have been attributed to FLRs driven by magnetospheric waveguide modes excited between the flankside magnetopause and internal turning points within the magnetosphere (e.g., Harrold and Samson, 1992; Samson et al., 1992b). These frequencies are denoted as CMS (cavity mode model of Samson et al.) frequencies. Recent observational studies found that the frequencies of stable Pc5 pulsations did not always have these discrete values (Ziesolleck and McDiarmid, 1995; Ziesolleck et al., 1998). In addition, space-based observational support for cavity/waveguide resonant modes is sparse (Waters et al., 2002). However, recent studies have reported that discrete oscillations in the Pc5 band can also be driven by solar wind dynamic pressure variations rather than by a magnetospheric waveguide mode (Kepko et al., 2002; Stephenson and Walker, 2002; Kepko and Spence, 2003).

Although a number of studies on Pc5 pulsations based on ground observations have been carried out, it is still unclear what processes play a significant role in determining the spectral properties of Pc5 pulsations on the ground. Ground-based observations alone cannot determine whether the observed ground signals are due to FLRs driven by compressional waves associated with the K-H waves or an external source (i.e., solar wind pressure variations) or due to direct transmission of the compressional fast mode wave to the ionosphere. In order to examine the above question, simultaneous ground and satellite observations within the FLR region are required. However, to the authors' knowledge, there have been few simultaneous ground-satellite observations of Pc5 at the resonance region (e.g., Potemra et al., 1989).

In this paper, we present simultaneous observations of Pc5 pulsations in the magnetosphere by the Polar and geosynchronous spacecraft and on the ground at the In- ternational Monitor for Auroral Geomagnetic Effects (IMAGE) array (Lühr et al., 1998) and the Sub-Auroral Magnetometer Network (SAMNET) (Yeoman et al., 1990). The event was observed from 0730 to 0810 UT on April 29, 2001. During this interval, ground-based observations in the morningside (0700-1000 local times) showed the FLR signatures and Polar on the morningside did encounter the FLR region. We make a direct comparison between the properties of Pc5 pulsations in space and on the ground. We also discuss a possible source for the Pc5 event using solar wind data from ACE and Cluster and magnetospheric data from GOES-8 and LANL-01A geosynchronous spacecraft.

The organization of this paper is as follows. In Section 2 we briefly describe the data sets used in this study. Section 3 presents the observations and describes data analysis. In Section 4 we discuss the relation between the Pc5 pulsations in the magnetosphere and on the ground. Section 5 presents the conclusions.

\section{Data Set}

Ground magnetic field data used in this study come from 33 magnetometers constituting the IMAGE and SAMNET magnetometer arrays. During the pulsation event studied here both IMAGE and SAMNET arrays were located on the morningside (0700-1000 local times). The geographic and corrected geomagnetic (CGM) coordinates of the magnetometer stations are listed in Tables 1 and 2 . The time resolution of the magnetometer data is $10 \mathrm{~s}$ for the IMAGE stations and $5 \mathrm{~s}$ for the SAMNET stations. The SAMNET data were resampled at 10 -s intervals after averaging the original 5-s data.

We compare the geomagnetic field data with the spin averaged ( $\sim 6 \mathrm{~s}$ ) electric field (Harvey et al., 1995) and magnetic field (Russell et al., 1995) data from Polar, which was located in the morningside magnetosphere. We also use the 
Table 2. List of the SAMNET Stations.

\begin{tabular}{|c|c|c|c|c|c|}
\hline \multicolumn{2}{|c|}{ Stations } & \multicolumn{2}{|c|}{ Geographic } & \multicolumn{2}{|c|}{ Corrected Geomagnetic } \\
\hline Name & Code & Latitude & Longitude & Latitude & Longitude \\
\hline$\overline{\text { Oulu }}$ & OUL & 65.10 & 25.85 & 61.63 & 105.48 \\
\hline Nordli & NOR & 64.37 & 13.36 & 61.50 & 94.98 \\
\hline Oulujärvi & OUJ & 64.52 & 27.23 & 60.98 & 106.31 \\
\hline Faroes & FAR & 62.05 & 352.98 & 60.73 & 77.52 \\
\hline Hankasalmi & HAN & 62.30 & 26.65 & 58.71 & 104.77 \\
\hline Lerwick & LER & 60.13 & 358.82 & 58.00 & 81.11 \\
\hline Nurmijärvi & NUR & 60.50 & 24.65 & 56.89 & 102.32 \\
\hline Uppsala & UPS & 59.90 & 17.35 & 56.52 & 95.96 \\
\hline Kvistaberg & KVI & 59.50 & 17.63 & 56.08 & 96.03 \\
\hline Glenmore & GML & 57.16 & 356.32 & 54.88 & 77.73 \\
\hline Borok & BOR & 58.03 & 38.33 & 54.05 & 113.41 \\
\hline York & YOR & 53.95 & 358.95 & 50.90 & 78.57 \\
\hline Hartland & HAD & 50.99 & 355.52 & 47.63 & 74.80 \\
\hline
\end{tabular}

magnetic field data from the geostationary GOES-8 satellite (Singer et al., 1996) in the post-midnight local time sector. The GOES-8 magnetic field data $(0.512 \mathrm{~s})$ were resampled at 6-s interval after boxcar averaging to compare to the field variations at Polar. The Polar and GOES-8 magnetic field data are expressed in mean-field-aligned (MFA) coordinates to separate the field perturbations into the transverse and compressional components. In this system the mean field is defined as the 600-s running vector averages $\langle\mathbf{B}\rangle$ of the $6 \mathrm{~s}$ magnetic field data $\mathbf{B}, e_{Z}$ is parallel to $\langle\mathbf{B}\rangle, e_{X}$ is perpendicular to $\langle\mathbf{B}\rangle$ and is radially outward, and $e_{Y}$ is also perpendicular to $\langle\mathbf{B}\rangle$ but is directed eastward. The transverse components $b_{X}$ and $b_{Y}$ are high-pass filtered as a result of the transformation into field aligned co-ordinates, and the parallel component defined by $b_{Z}=|\mathbf{B}-\langle\mathbf{B}\rangle|$ is the highpass filtered compressional component. The Polar electric field data measured on the spin plane are rotated into MFA coordinates. We use the particle flux data obtained from the Los Alamos National Laboratory (LANL) geosynchronous spacecraft measurements. The particle flux variations are compared with the magnetic field perturbations observed at Polar and GOES-8.

The solar wind and interplanetary magnetic field (IMF) data was measured by the ACE satellite. The key parameter ACE data was obtained from the CDAWeb data service (http://cdaweb.gsfc.nasa.gov/). The time resolution is $64 \mathrm{~s}$ for the plasma parameters and $16 \mathrm{~s}$ for the magnetic field. The spin averaged ( $\sim \mathrm{s}$ ) spacecraft potential (Gustafsson et al., 1997) and magnetic field (Balogh et al., 2001) data from Cluster near the bow shock was used to monitor the solar wind dynamic pressure variation closer to the Earth.

\section{Observations}

\subsection{Solar wind observations}

Figures 1(a) to $1(\mathrm{~g})$ show the solar wind density, velocity, dynamic pressure, and the interplanetary magnetic field (IMF) in the GSE (geocentric solar ecliptic) coordinates observed by the ACE spacecraft located near GSE $(x, y, z) \sim(223.6,17.4,-23.9) R_{E}$ for the interval from 0700 to 0900 UT on April 29, 2001. The ACE data was lagged by a constant time delay of $39 \mathrm{~min}$ for comparison with the Cluster data measured just inside the bow shock. The solar wind speed gradually changed from 640 to 610 $\mathrm{km} / \mathrm{s}$. The solar wind density increased from 4 to $7 \mathrm{~cm}^{-3}$ at $\sim 0743$ UT and then gradually decreased for about 20 min to reach the level prior to $\sim 0743$ UT. The density then rose at 0825 UT and continued to do so until the end of this interval. The solar wind dynamic pressure variations mainly corresponded to variations in the solar wind density. The IMF was directed earthward $\left(B_{X}<0\right)$ and duskward $\left(B_{Y}>0\right)$ and was nearly parallel to the solar ecliptic plane except for the three intervals, 0700-0708 UT, 0725-0800 UT, and 0845-0900 UT.

The spin-averaged $(\sim 4 \mathrm{~s})$ spacecraft potential and $B_{Z}$ magnetic field data from spacecraft 1 of Cluster are plotted in Figs. 1(h) and 1(i). Note that the other Cluster spacecraft observed nearly identical signatures (not shown). During the 2-hour interval, 0700-0900 UT, Cluster was near the north/morningside bow shock and moved from GSE $(x, y, z) \sim(7.9,-6.2,9.0)$ to $(8.9,-8.1,8.7) R_{E}$. The spacecraft potential was about -5 to $-6 \mathrm{~V}$ from 0700 to $0743 \mathrm{UT}$. We approximate a plasma density about 10 $\mathrm{cm}^{-3}$ from Fig. 3 of Pedersen et al. (2001). This indicates that Cluster was in the magnetosheath. Turbulent magnetosheath magnetic field variations were observed by Cluster for this interval. Cluster entered the solar wind at around 0743 UT. The solar wind entries (i.e., bow shock crossings) can be clearly identified from the steplike increase in the spacecraft potential and decrease in $B_{Z}$. Cluster was mainly in the solar wind and briefly entered the magnetosheath several times during the interval 0743-0804 UT. This time period corresponds to the interval of the solar wind dynamic pressure increase observed at ACE. Thus, Cluster's entry into the solar wind from the magnetosheath during the interval of 0743-0804 UT was due to a solar wind pressure increase. Cluster moved back into the magnetosheath from 0804 to 0829 UT and then entered and stayed in the solar wind for the remainder of the interval. This solar wind entry was due to the solar wind pressure increase and/or satellite's outward orbital motion. Cluster observed highly variable IMF in the solar wind from 0829 to 0850 UT. This indicates that the IMF connected the spacecraft to the bow shock. 

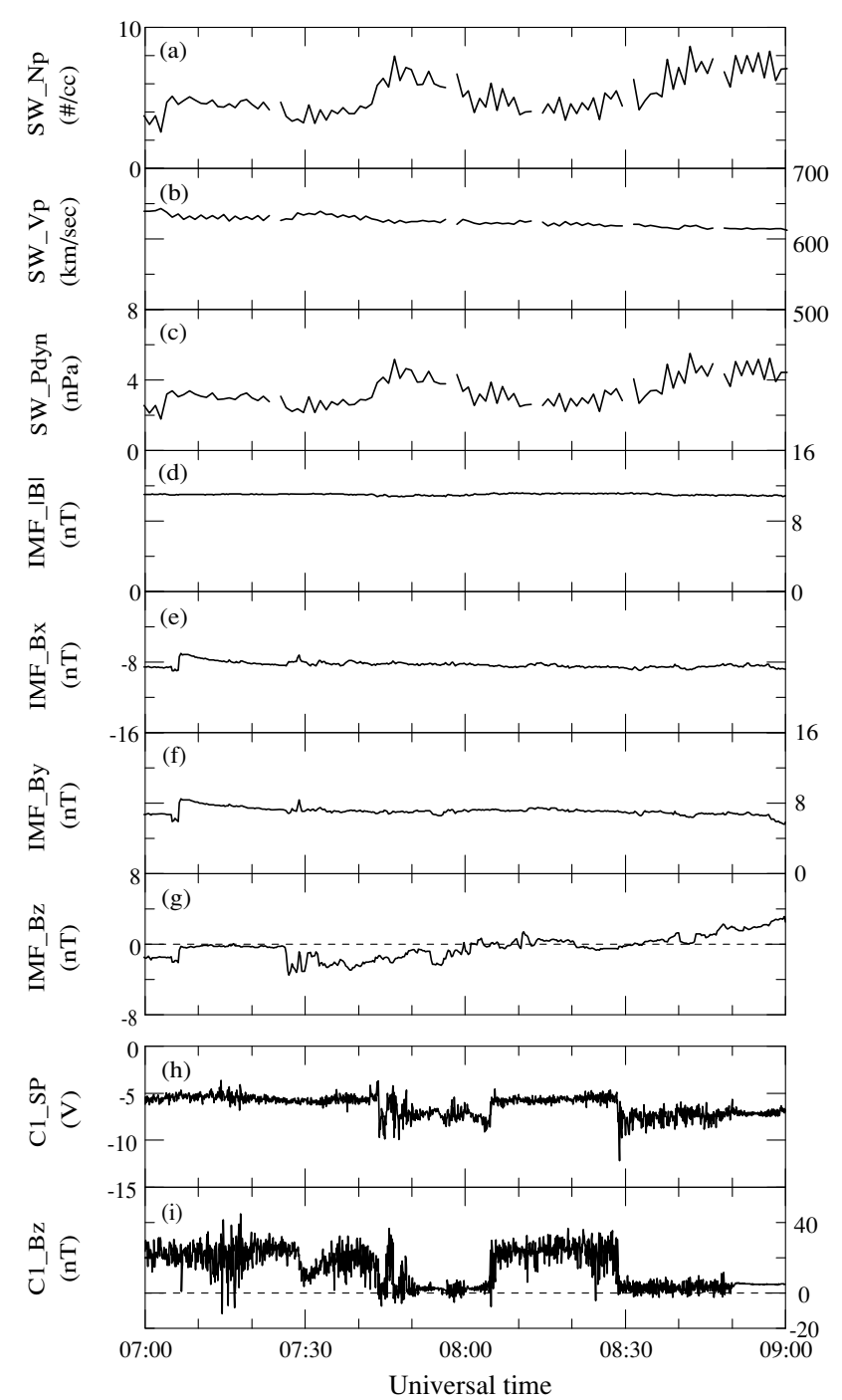

Fig. 1. Solar wind conditions observed by ACE and Cluster during 0700-0900 UT on April 29 (doy 119), 2001. From (a) to (i), the panels show solar wind density, velocity, dynamic pressure, and interplanetary magnetic fields observed by ACE, and the spacecraft potential and magnetic $B_{Z}$ component observed by Cluster, respectively. ACE data were lagged $39 \mathrm{~min}$ for comparison to the Cluster data.

That is, Cluster had entered the foreshock (e.g., Hoppe et al., 1981). After 0850 UT Cluster was completely in "pristine" solar wind.

\subsection{Magnetospheric observations}

In this section, we investigate the observations of the Polar, LANL-01A, and GOES-8 satellites in the magnetosphere. Figure 2 shows the positions of the three satellites projected onto the $\mathrm{XY}_{\mathrm{GSE}}$ plane at $0750 \mathrm{UT}$. The model magnetopause with solar wind dynamic pressure $P_{S W}=5$ $\mathrm{nPa}$ and IMF $B_{Z}=-1 \mathrm{nT}$ (Shue et al., 1998) is plotted with the solid curve for reference.

During the interval 0700-0900 UT, Polar was located near the magnetic equator with a magnetic latitude between $-11^{\circ}$ and $6.6^{\circ}$ on the morningside (9.6-9.8 MLT), crossing the $L$ shells between 7.5 and 8.8 and moving from 7.3 to $8.7 R_{E}$ in the radial distance. Figures 3(a) and 3(b) show the electron density estimated from the spacecraft potential (Scudder et al., 2000) and the magnetic field magnitude

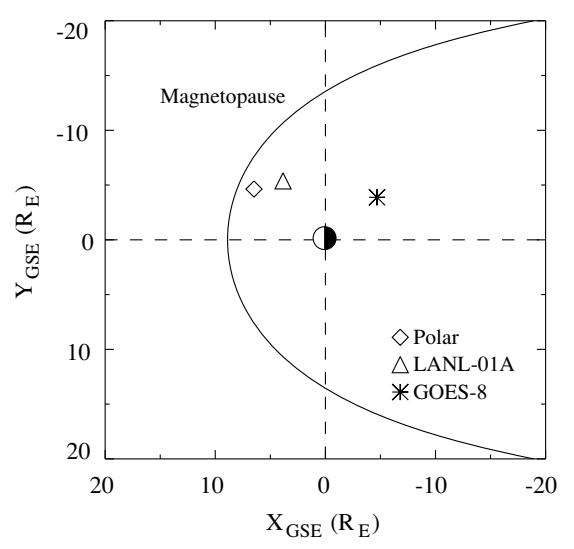

Fig. 2. Locations of the magnetopause and satellites at 0750 UT on April 29 (doy 119), 2001. The magnetopause location is predicted using the Shue et al. (1998) model with $P_{S W}=5 \mathrm{nPa}$ and IMF $B_{Z}=-1 \mathrm{nT}$.

at Polar and the spacecraft potential from Cluster, respectively. Polar observed a gradual decrease in the average magnetic field strength from 0700 to 0820 UT. For the interval $\sim 0820-0900$ UT, Polar was still on the outbound pass and observed a gradual increase in $B_{T}$. Such a field increase may have been due to the gradual compression of the magnetosphere by an increase in the solar wind dynamic pressure (see Fig. 1). There were also quasi-periodic changes in the electron density and $B_{T}$. Although the density fluctuations were more complex than the $B_{T}$ perturbations, we found that the peaks in the $B_{T}$ oscillated in phase with the density fluctuations. This relationship between the $B_{T}$ and plasma density can be explained in terms of a fast mode signature.

In order to find the source of the magnetospheric perturbations, the spacecraft potential at Cluster and $B_{T}$ at Polar are compared in Fig. 3(b). During the brief solar wind entries marked by solid circles and density enhancements in the magnetosheath and solar wind marked by arrows, the magnetic field at Polar was enhanced. The $B_{T}$ enhancement at 0830 UT occurred at the time when Cluster entered the solar wind from the magnetosheath. During the interval from $\sim 0805$ to $\sim 0827 \mathrm{UT}$, there are no significant $B_{T}$ and spacecraft potential variations. These observations indicate that the magnetospheric perturbations are associated with the motion of the magnetopause and the bow shock, which is driven by solar wind dynamic pressure variations.

Figures 3(c) to 3(f) show three magnetic field components $\left(b_{X}, b_{Y}\right.$, and $\left.b_{Z}\right)$ and the radial electric field component $\left(E_{X}\right)$, respectively. The $\sim 6$-min oscillations are clear in $E_{X}$ from 0700 to 0815 UT. Visual inspection of the perturbations in $b_{Y}$ and $E_{X}$ components during the interval from 0700 to 0740 indicates that $E_{X}$ oscillated $\sim 90^{\circ}$ out of phase with $b_{Y}$. The amplitude of the $E_{X}$ oscillation was enhanced during the interval $\sim 0745-0815$ UT, whereas the $\sim 6$-min oscillation in $b_{Y}$ disappeared around 0800 UT. Note that MLAT of Polar was $\sim 0^{\circ}$ at $\sim 0800$ UT. The spacecraft was near the magnetic equator so it missed the magnetic field signature of the fundamental mode standing wave while detecting the second harmonic. Since standing Alfvén waves are characterized by field perturbations in 


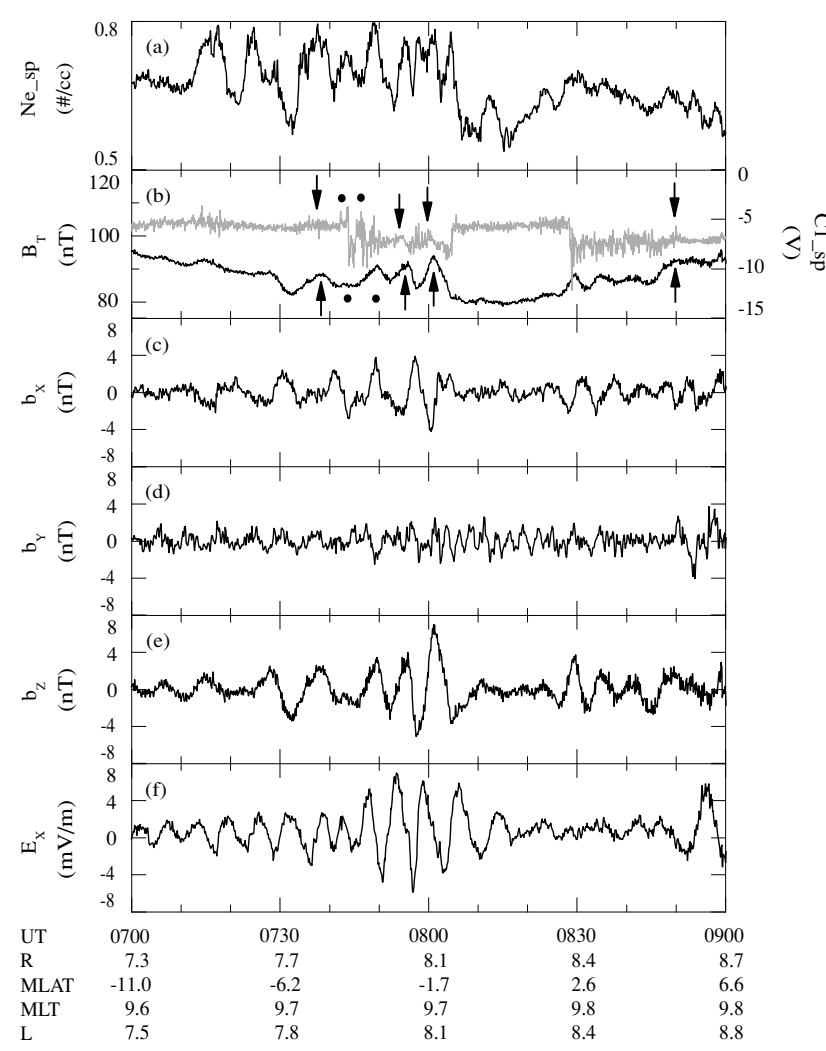

Fig. 3. Polar data during 0700-0900 UT on April 29 (doy 119), 2001. From (a) to (e) the panels show magnetospheric density from the spacecraft potential (SP), magnetic field magnitude, perturbations of the compressional and azimuthal magnetic field $b_{Z}$ and $b_{Y}$, and radial electric field $E_{X}$, respectively. The spacecraft potential data from Cluster is also shown in panel (b).

the azimuthal magnetic field and radial electric field with a $90^{\circ} / 270^{\circ}$ phase shift, $\sim 6$-min oscillations in $b_{Y}$ and $E_{X}$ would be consistent with those of a fundamental standing Alfvén wave.

If a fast mode wave excites a standing Alfvén wave in the manner described by the standard theory of field line resonance (Chen and Hasegawa, 1974; Southwood, 1974), the perturbations in $E_{X}$ and $b_{Z}$ should be very coherent. Figure 4 shows the coherence between $E_{X}$ and $b_{Z}$ for the interval 0710-0820 UT. As expected from the time series plots in Fig. 3, $E_{X}$ and $b_{Z}$ produce spectral enhancements in the $2-3 \mathrm{mHz}$ band with a central frequency at $\sim 2.8 \mathrm{mHz}$, with the coherence at this frequency greater than 0.6. This suggests that a coupling between $E_{X}$ and $b_{Z}$ occurred at or near the Polar location. Also, there are several frequencies which coherence is greater than that of $2.8 \mathrm{mHz}(5,7$, and $11 \mathrm{mHz}$ ). However, we investigate $2.8 \mathrm{mHz}$ pulsations in this study because their spectral power densities are lower by a factor of 10 than that of $2.8 \mathrm{mHz}$.

During the period of interest, geosynchronous satellites LANL-01A and GOES-8 were located on the morningside and in the post-midnight magnetosphere, respectively (see Fig. 2). Figure 5 shows $b_{Z}$ at Polar, energetic particle fluxes at LANL-01A, and $b_{Z}$ at GOES-8. The magnetic local time in each panel indicates the location of the satellite at 0750 UT. Polar and LANL-01A data show nearly identical perturbations marked by arrows. This indicates that the particle
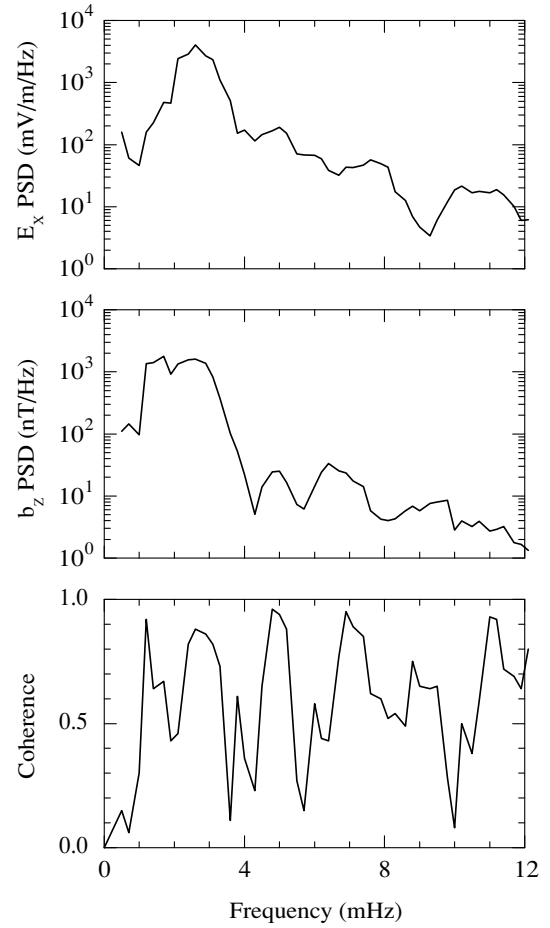

Fig. 4. The power spectral densities of $E_{X}$ and $b_{Z}$ and the coherence between $E_{X}$ and $b_{Z}$ of Polar for the time interval 0710-0820 UT on April 29 (doy 119), 2001.

flux enhancement is due to the magnetic field compression which causes adiabatic acceleration. GOES- 8 observed a compressional magnetic field perturbation. Its amplitude was enhanced near 0800 UT. Although Polar and GOES-8 were separated by as much as $\sim 7$ hours in local time, the three perturbations at GOES- 8 near 0800 UT indicated by arrows are similar in amplitude modulation to those at Polar. The amplitude of the perturbations at GOES- 8 was smaller than those at Polar. This indicates that Polar was closer to the source region of the oscillations than GOES-8. The Polar perturbations led the GOES- 8 perturbations by $\sim 90 \mathrm{~s}$. Since the two satellites are radially separated by about $\sim 11$ $R_{E}$, the propagation speed is $\sim 800 \mathrm{~km} / \mathrm{s}$. This velocity is comparable to the equatorial Alfvén velocity estimated by Takahashi and Anderson (1992). Since the Alfvén velocity is close to the magnetosonic speed near geosynchronous orbit, we suggest that the perturbations are excited in the dayside by fast mode waves propagating tailward.

\subsection{Ground-based measurements}

Figure 6 shows the geographic locations of the 33 morningside ground stations and the ionospheric footprints of the Polar satellite from 0700 to 0900 UT on April 29, 2001. The satellite's footprint during the 2-hour interval is plotted with a solid line and marked every 30 minutes by the open circles. The footprints of the satellite were calculated using the magnetic field model of Tsyganenko (1989).

Figure 7 shows a comparison of the spacecraft potential at Cluster and the $H$ component of SOR. The geomagnetic field data has been filtered by removing the 500-s running averages from the $H$ component. It is clear that strongly enhanced Pc5 (period $=\sim 6 \mathrm{~min}$ ) oscillations occurred during an interval from $\sim 0740$ to $\sim 0805$ UT when Cluster experi- 


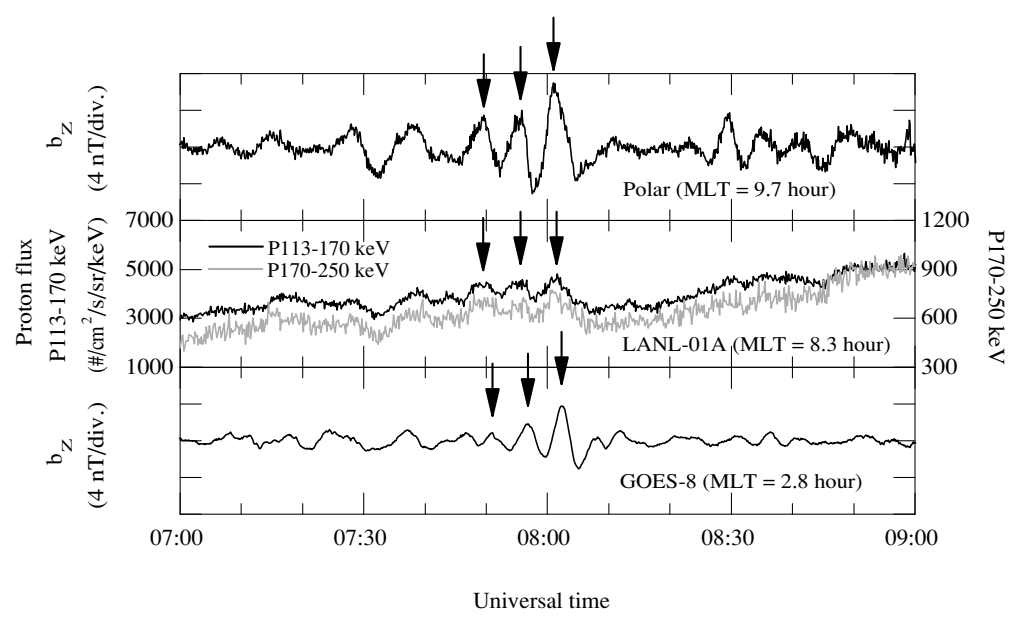

Fig. 5. Magnetic field and energetic proton flux data from Polar, LANL-01A, and GOES-8. The locations of three satellites are given in magnetic local time at 0750 UT on April 29 (doy 119), 2001.

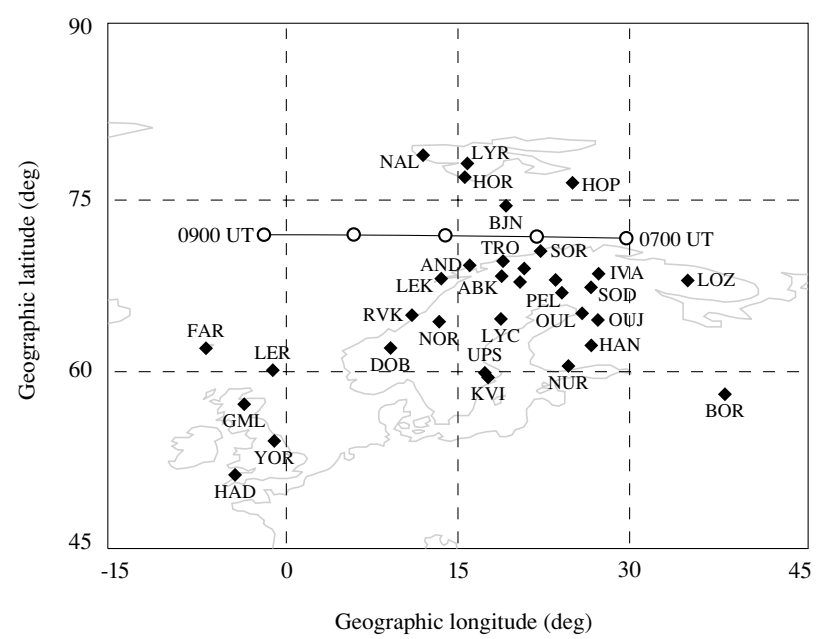

Fig. 6. The geographic locations of 33 magnetometers of the IMAGE and SAMNET arrays (see Tables 1 and 2 for details of station coordinates). The solid line shows the footprint trace of the Polar spacecraft from 0700 to 0900 UT on April 29 (doy 119), 2001 and is marked every 30 minutes by the open circles.

enced transient bow shock crossings and observed enhanced solar wind density increases. The $\sim 6$-min oscillations disappeared as Cluster re-entered the magnetosheath from the solar wind at $\sim 0805$ UT. This indicates that the enhanced geomagnetic oscillations at SOR are associated with the external variations.

Figure 8 shows the geomagnetic field $H$ and $D$ components for 0720-0820 UT at the 33 stations from the SAMNET and IMAGE magnetometer network. The magnetic latitude and local time at the center (0750 UT) of the 1hour interval are shown to the right of each magnetic field trace. In each panel, stations are ordered from high-latitude at the top to low-latitude at the bottom. The vertical dotted lines indicate the peaks of the $H$ component at SOR.

The $H$ and $D$ components at all stations clearly show enhanced Pc5 oscillations with a period of $\sim 6$ min during the interval from $\sim 0740$ to $\sim 0800$ UT. From the multistation data, we can find the spatial and temporal properties
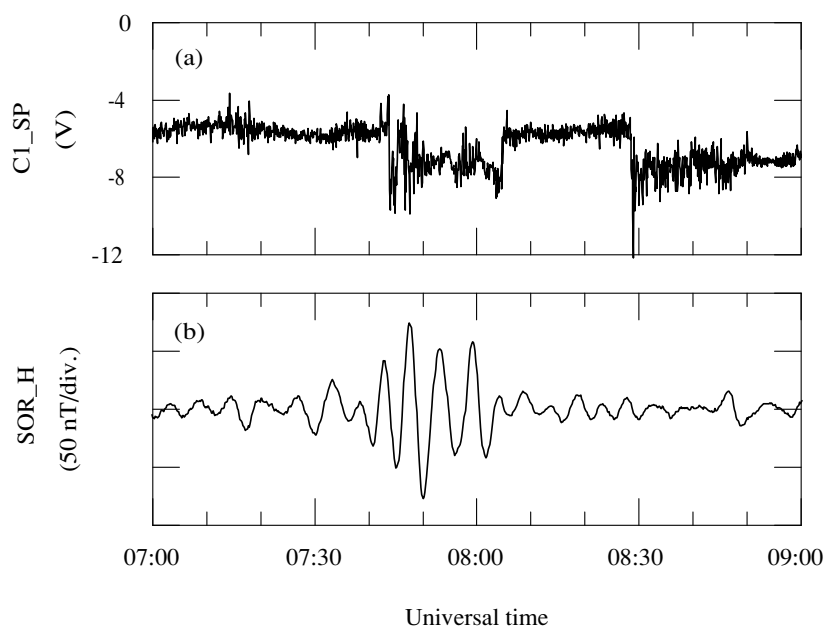

Fig. 7. Spacecraft potential observed by Cluster, and the $H$ component of the geomagnetic field at SOR during 0700-0900 UT on April 29 (doy 119), 2001.

of the Pc5 oscillations. First, the $H$ perturbations at SOR were almost out of phase with those at BJN, while the $D$ component was not. Phase reversal in the $H$ component occurred between $\sim 67^{\circ}$ and $\sim 71^{\circ}$. The amplitude of the $H$ component oscillation was maximum at SOR. Second, the $H$ component oscillations at GML $\left(\mathrm{CGM}=54.88^{\circ}\right.$, $\mathrm{LT}=7.6$ hours $)$ lagged behind those at BOR $\left(54.05^{\circ}, 10.4\right.$ hours). This suggests that Pc5 oscillations have a phase change with longitude. Third, the relative amplitude and phases between the $H$ and $D$ components differed from station to station, possibly depending on the station location relative to the source region of the oscillation.

We applied the cross-spectral analysis technique (Takahashi et al., 2001) to the ground magnetometer data for the interval from 0735 to 0805 UT, to determine the spatial variation of the amplitude, phase, and inter-station coherence of the Pc5 event. Figure 9 shows the stacked plots of the power spectra of the $H$ components from selected stations and covering magnetic latitudes from $76^{\circ}$ to $48^{\circ}$. As expected from the time series plots of Fig. 8, the selected 

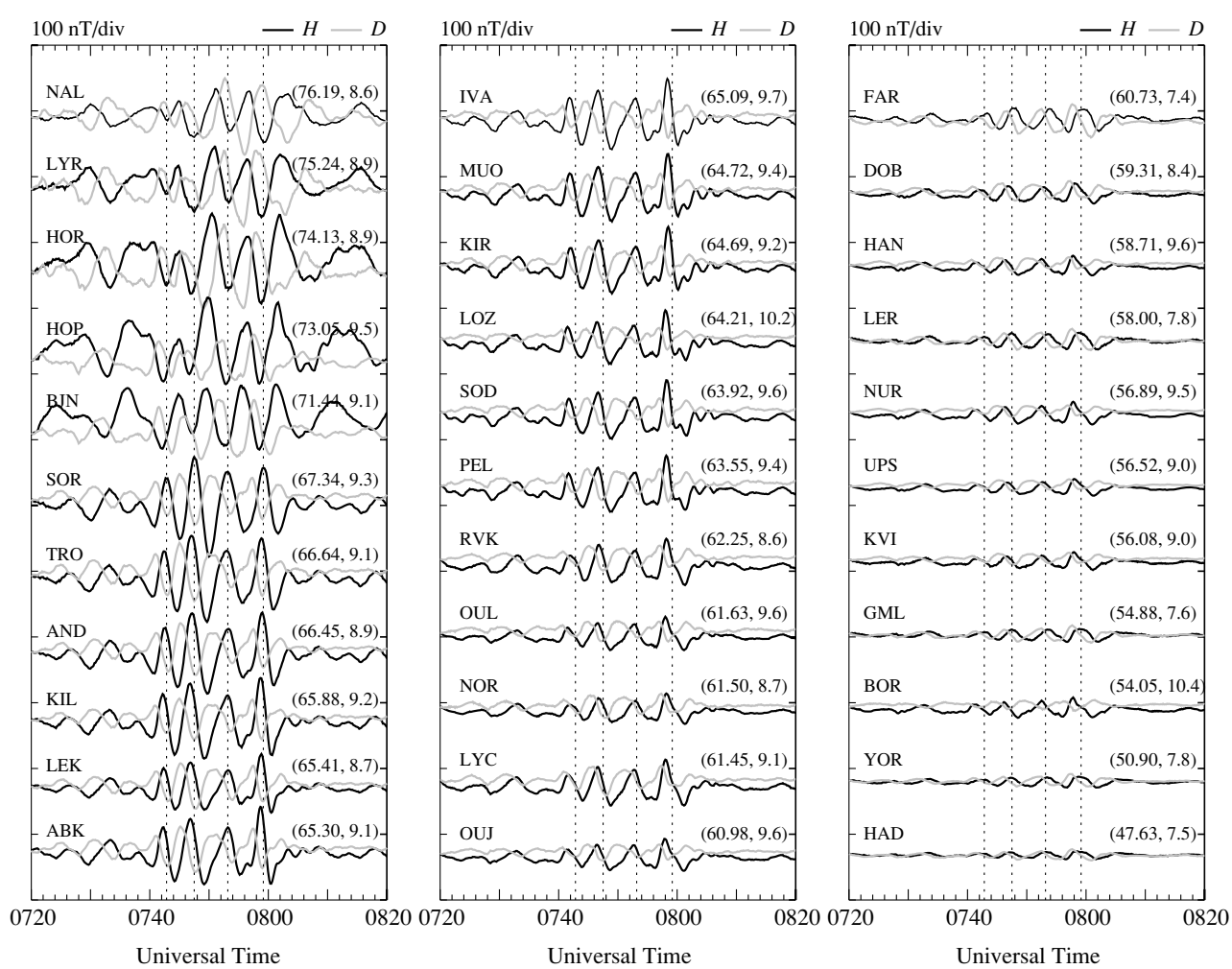

Fig. 8. The $H$ (black) and $D$ (gray) components of geomagnetic field measured by the 33 magnetometers during 0720-0820 UT on April 29 (doy 119), 2001. All three panels have the same scales of $100 \mathrm{nT} / \mathrm{div}$. The vertical lines are at 0742:50, 0747:30, 0753:10, and 0759:10 UT, respectively.

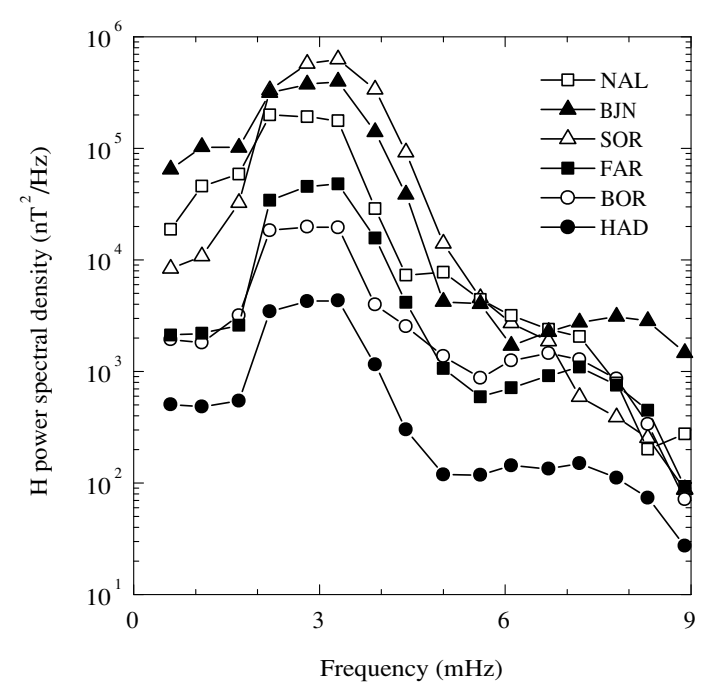

Fig. 9. The power spectrum of the $H$ component from the six selected stations from higher to lower latitudes for the interval 0735-0805 UT on April 29 (doy 119), 2001.

stations showed an almost identical spectral shape, with a spectral peak centered at $2.8 \mathrm{mHz}$ (period $\sim 6 \mathrm{~min}$ ). Note that the $H$ and $D$ components at all ground stations had a very similar spectral enhancement and a central frequency of $2.8 \mathrm{mHz}$ (not shown here). This latitude-independent frequency was used for the coherence and cross-phase calculations.

Figure 10(a) shows the coherence between SOR and other stations. A coherence of more than 0.7 occurred from

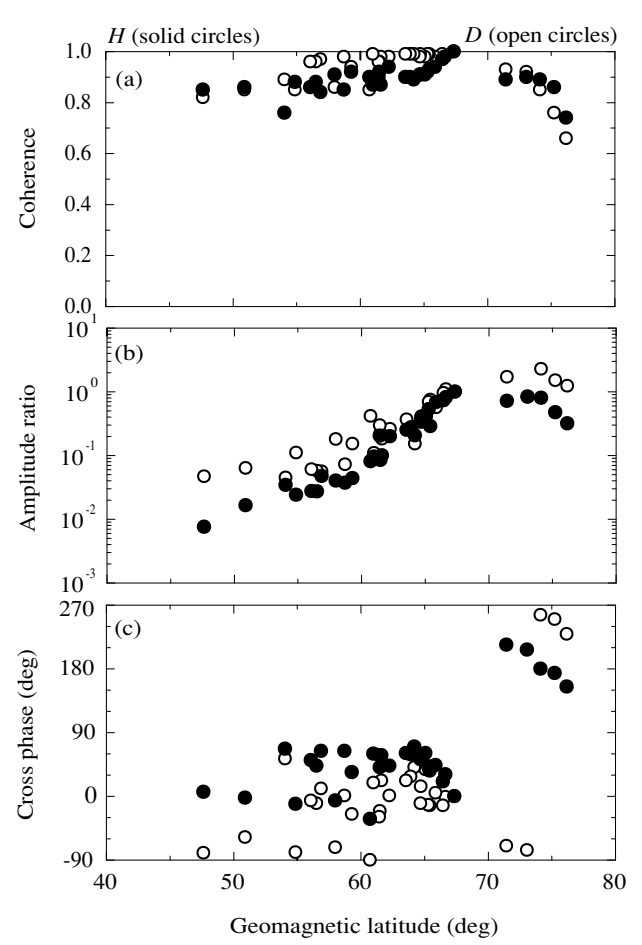

Fig. 10. The coherence coefficients (a), power ratio (b), and cross-phase (c) variations between $\mathrm{SOR}$ and other stations at $2.8 \mathrm{mHz}$ as a function of geomagnetic latitude. The solid (open) circles represent the $H(D)$ component.

high- $\left(\mathrm{CGM}=75^{\circ}\right)$ to low- $\left(<50^{\circ}\right)$ latitudes for the $H$ and $D$ components, indicating that the Pc5 oscillations were excited by a common source mechanism throughout the ob- 


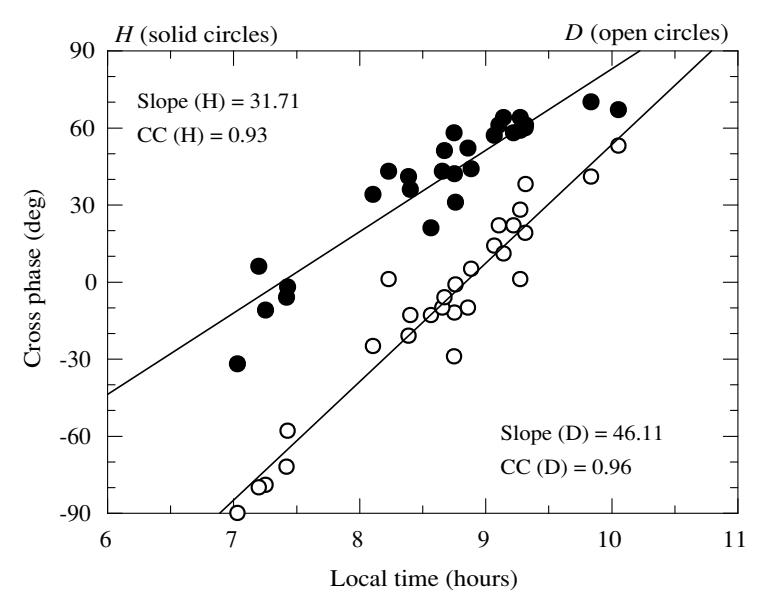

Fig. 11. The cross phase variations between SOR and other stations at 2.8 $\mathrm{mHz}$ as a function of local time. The solid (open) circles represent the $H(D)$ component and two lines give the result of the line fitting. The slopes of the lines and the linear correlation coefficients (CC) are also given.

served latitude range. The latitude profile of the amplitude ratio, i.e., the ratio normalized by the $H$ and $D$ components at SOR, is shown in Fig. 10(b). The normalized amplitude and cross-phase (Fig. 10(c)) were calculated only if the coherence was higher than 0.7. The normalized amplitudes in $H$ and $D$ increased in latitude until $\sim 67^{\circ}$, before decreasing once again. The amplitude maximum was near $67^{\circ}$, indicating that the field line is connected to the source region or to the region of a strong mode conversion (i.e., the FLR region) (Chen and Hasegawa, 1974; Southwood, 1974).

The cross-phase of the $H$ component exhibits two groups, depending on latitude. One is distributed throughout the range from $-30^{\circ}$ to $90^{\circ}$ at $\mathrm{CGM}<67^{\circ}$ and the other group between the range of $150^{\circ}$ and $210^{\circ}$ at $\mathrm{CGM}>67^{\circ}$. The maximum amplitude was located near the boundary between the two groups. Such a phase reversal at the maximum amplitude location is similar to that in previous studies (e.g., Samson and Rostoker, 1972; Walker et al., 1979; Mathie et al., 1999) and was interpreted as an FLR signature. The cross-phase data points were considerably scattered along magnetic latitude. That is, the data points over the range of latitudes did not fall exactly on $0^{\circ}$ or $180^{\circ}$.

As mentioned above, the Pc5 oscillation had a phase variation in the azimuthal direction, suggesting that the scatter in the cross-phase could have been due to a longitudinal phase variation. Figure 11 shows the cross-phase versus local time for the $H$ (solid circles) and $D$ (open circles) components. We included the slopes as determined by leastsquares fitting and the linear correlation coefficient (CC). Although the $H$ and $D$ components had different slopes, it is clear that the cross-phase for both components strongly depends on local time. The cross-phase increased with local time, implying that its propagation direction was away from noon (i.e., a westward propagation). The phase difference between 10 and 7 LT was $\sim 95^{\circ}\left(\sim 138^{\circ}\right)$ for $H$ (D), corresponding to a time difference of $\sim 94 \mathrm{~s}(\sim 137 \mathrm{~s})$. That is, the phase speed was $\sim 29^{\circ}$ in longitude per minute $\left(\sim 20^{\circ}\right.$ in longitude per minute) for $H(D)$. If we assume that the Pc5 oscillations were those perturbations associated
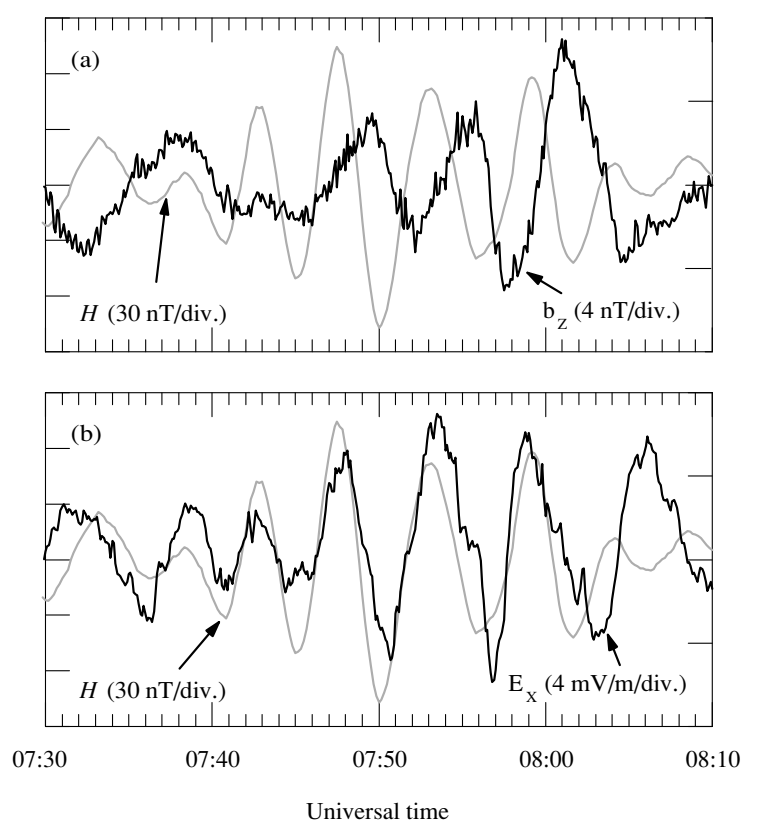

Fig. 12. Compressional magnetic field $\left(b_{Z}\right)$ and radial electric field $\left(E_{X}\right)$ perturbations at Polar and the $H$ component magnetic field variations at SOR for the interval 0730-0810 UT on April 29 (doy 119), 2001.

with FLR at $L \sim 8.1$ (i.e., Polar's location), the azimuthal propagation speed mapped to the magnetospheric equator at $L \sim 8.1$ would be $\sim 430 \mathrm{~km} / \mathrm{s}$ for $H$ and $\sim 300 \mathrm{~km} / \mathrm{s}$ for $D$, respectively. The reason for the discrepancy between the azimuthal phase speeds of the $H$ and $D$ components is unclear. A possible interpretation is that the polarization of the ground signal corresponding to a magnetospheric wave is not constant over the observed latitude-longitude range.

Ground-based magnetometers do not directly detect the corresponding magnetic field pulsations in space. That is, they measure magnetic fields whose sources are distributed in the ionosphere. Therefore spacecraft observations are necessary to examine how ground magnetic field variations are related to the magnetic field oscillations in space. As shown in Fig. 6, we have a very nice ground-satellite conjunction where a FLR signature was observed. In order to compare the magnetic field variations on the ground and in space near the FLR region, the compressional magnetic field $\left(b_{Z}\right)$ and radial electric field $\left(E_{X}\right)$ at Polar and the $H$ component at SOR, which is located in the conjugate point of Polar, are shown in Fig. 12. In Fig. 12(a) the positive peaks of $H$ at 0747:30, 0753:10, and 0759:10 UT lead the $b_{Z}$ peaks by $\sim 2 \mathrm{~min}$. Since Polar was near the conjugate point of SOR, we do not attribute the observed time lag to radial and/or azimuthal wave propagation. We find that the $H$ variations are roughly in quadrature relationship with the $b_{Z}$ variations with the peaks at $0749: 36,0755: 24$, and 0801:00 UT. It seems that the $H$ component has a positive (negative) peak before each interval of magnetic field compression (rarefaction). Figure 12(b) shows that the SOR $H$ component has a great similarity to the perturbations in $E_{X}$ from 0740 to $0800 \mathrm{UT}$. This implies that the ground perturbations are associated with the transverse Alfvén mode rather than the compressional mode.

It has been shown previously that shear Alfvén waves 

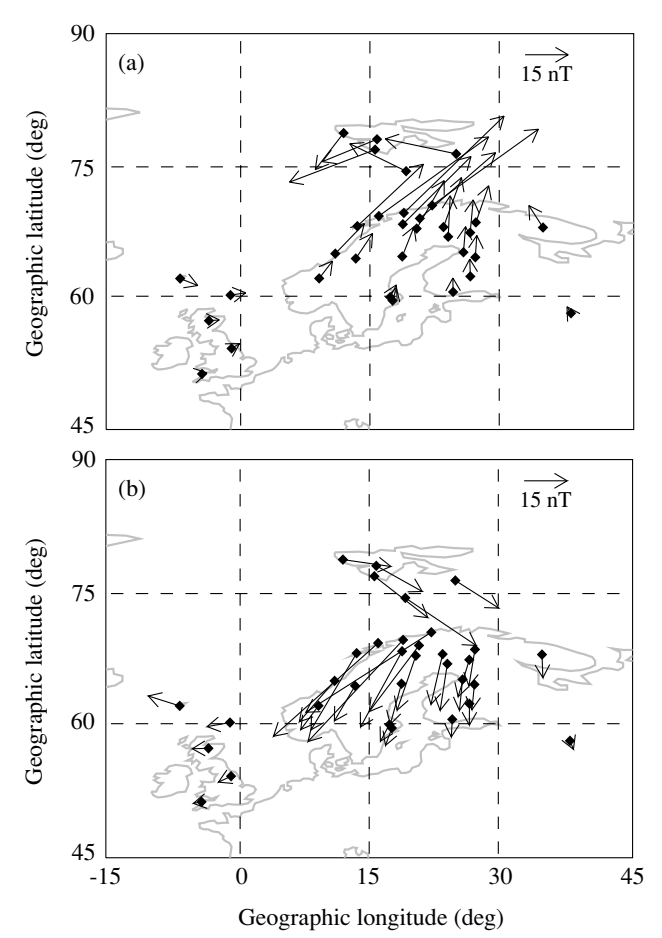

Fig. 13. Snapshots of the equivalent ionospheric current patterns of the long period geomagnetic pulsations at 0742:50 UT (a) and 0745:10 UT (b) on April 29 (doy 119), 2001. The arrows are equivalent current vectors in nano-Tesla.

carry field-aligned currents and that ground geomagnetic perturbations are due to the ionospheric Hall current, which is generated by field-aligned currents (e.g., Walker et al., 1979; Greenwald and Walker, 1980). If the Pc5 geomagnetic pulsations in our study were associated with a shear Alfvén mode, there should be an ionospheric current vortex centered at the region of the resonant field line (around $70^{\circ}$ geographic latitude). To investigate the ionospheric current pattern, the horizontal band-passed $(2-5 \mathrm{mHz})$ magnetic perturbation vectors were rotated by $90^{\circ}$ in the clockwise direction. Figures 13(a) and 13(b), which present a snapshot of equivalent current vectors at 0742:50 UT and 0745:10 UT, respectively, corresponding to the times of the positive and negative peaks for the first cycle of the oscillation at SOR, are consistent with a vortical equivalent ionospheric current structure centered around $70^{\circ}$. The rotational sense of the current vortex was counterclockwise (clockwise) at the positive (negative) peak, consistent with a source of Pc5 pulsations due to an oscillation of the ionospheric Hall currents generated by the shear Alfvén waves.

Besides the mode coupling, there is another mechanism for field-aligned current generation. Kataoka et al. (2004) suggested that the source current of the field-aligned current is the inertial current associated with in-out plasma acceleration in the magnetosphere near the impact region. Pairs of field-aligned currents drive traveling convection vortices (TCVs). However, TCVs are highly localized in longitude with a spatial scale of 1000-2000 km encompassing several hours in local time. The Pc5 event in this paper is more similar to that of Motoba et al. (2002) in period and the location of the ionospheric current vortex. They also suggested that a pair of field-aligned currents is generated well inside the magnetosphere through mode conversion from the compressional to Alfvén modes. However, they did not investigate the mode conversion in the magnetosphere. In this study, we analyzed simultaneous observations of Pc5 pulsations on the ground and in space and found evidence of mode conversion in the magnetosphere.

\section{Discussion and Conclusions}

We presented a detailed case study of Pc5 pulsations observed at IMAGE and SAMNET stations on the morningside. The latitudinal structure of the phase and amplitude of pulsations is consistent with those reported in previous studies (e.g., Samson and Rostoker, 1972; Walker et al., 1979; Mathie et al., 1999), which interpreted the waves as FLR driven by fast-mode waves generated by the K-H instability at the magnetopause or by a magnetospheric waveguide mode. Since the previous studies mainly used ground-based data, it was difficult to discuss how the magnetic field oscillations in space are related to the ground signals. Our study showed that the ground perturbations are caused by oscillations of the ionospheric currents generated by the shear Alfvén waves coupled with the compressional oscillations and that the compressional perturbations are caused by the solar wind pressure variations.

One would expect that the latitude-independent Pc5 pulsations are directly related to the compressional waves launched at the wavy magnetopause. Such waves propagate radially from the source region. If the ground Pc5 pulsations result from radially propagating fast mode waves, the phase would change linearly with $L$ or the magnetic latitude. However, the phase variations of the ground perturbations did not show a linear variation with the magnetic latitude (see Fig. 10). Thus, the ground Pc5 pulsations cannot be explained by radially propagating waves.

We have to explain why the compressional oscillations observed at Polar cannot propagate into high-latitude ground stations. Previous observations showed that compressional waves propagating across magnetic shells are mainly found near the equatorial region (Yumoto et al., 1985; Takahashi and Anderson, 1992). These features were confirmed by the numerical simulation of Lee and Lysak (1994). Lee (1996) discussed the relationship between compressional wave propagation and its cutoff boundary in a dipole geometry and showed that the cutoff frequency of compressional waves increases as they propagate earthward and toward increasing magnetic latitude. This indicates that relatively lower frequency waves (i.e., Pc5 band) are strongly confined near the equatorial region in the outer magnetosphere and close to the magnetopause. That is, compressional Pc5 pulsations cannot propagate easily into high-latitude regions and deep into the magnetosphere.

We observed the azimuthal propagation of the Pc5 pulsations away from noon in the morningside (i.e., antisunward propagation). The azimuthal phase speed mapped to the magnetospheric equator at $L \sim 8.1$ is $\sim 300-430 \mathrm{~km} / \mathrm{s}$. This is much smaller than the fast mode speed $(\sim 800 \mathrm{~km} / \mathrm{s})$ propagating tailward. Thus, the observed azimuthal propagation speed cannot be explained in terms of tailward propagation of a fast mode wave. The observed azimuthal speed 
is $\sim 50-70 \%$ of solar wind speed $(\sim 600 \mathrm{~km} / \mathrm{s})$, which is in the range of the expected flow speed of the morningside magnetosheath (Spreiter et al., 1966). We have suggested above that the Pc5 geomagnetic oscillations on the ground are caused by an oscillation of the ionospheric currents generated by resonant Alfvén waves coupled to the compressional oscillations driven by external pressure variations at the magnetopause. This indicates that the azimuthal phase speed corresponds to a tailward moving speed of magnetopause perturbations. Similar observations were previously reported by Potemra et al. (1989).

We observed that the ground magnetometer data show a roughly quadrature signature during each magnetic compression in space. Since the shear Alfvén waves drive the ionospheric Hall current, we examined the ionospheric equivalent current at the positive and negative peaks of the oscillations at SOR. This indicates that a pair of fieldaligned currents, flowing upward in the west and downward in the east, occurred during compression of the magnetosphere. This feature is similar to that during SI (sudden impulse)/SSC (sudden storm commencement) events (e.g., Araki, 1994), but the magnetospheric compression in our case is localized and is due to wavy magnetopause motion. The ionospheric equivalent current system provides the information about the plasma convection pattern in the magnetospheric equatorial plane (Glassmeier et al., 1989). This implies that field-aligned currents carry the information about the magnetopause motion to the ground. As shown in Fig. 13, the equivalent current flow in the central part of the current system is directed toward higher latitudes. The corresponding plasma motion maps to a radial inward motion in the equatorial magnetospheric plane. Such plasma flow is expected near the inside of the magnetopause when the magnetopause moves inward.

We examined morningside Pc5 (period $=\sim 6 \mathrm{~min}$ ) pulsations that were observed simultaneously in space and on the ground. The phase and amplitude signatures of the Pc5 on the ground stations were consistent with previous FLR observations. Fortunately, the Polar spacecraft passed the FLR region and observed a coupling between externally driven compressional waves and shear Alfvén waves. We examined the relationship between the Pc5 oscillations in space and those on the ground and found that the ground Pc5 pulsations had a direct connection with shear Alfvén waves. We further showed that the ground $H$ and $D$ perturbations were due to field-aligned currents associated with shear Alfvén waves. The azimuthal phase speed of the Pc5 was comparable to the magnetosheath flow speed. This may be interpreted to mean that the source of the FLR propagated antisunward at a velocity comparable to the magnetosheath flow speed.

Acknowledgments. We thank C. T. Russell for the Polar magnetic field data. We also thank the Finnish Meteorological Institute which maintains the IMAGE magnetometer network. The authors thank the SAMNET team for magnetometer data. SAMNET is a PPARC National Facility operated by Lancaster University. The key parameter data from ACE were provided by the NASA/GSFC data processing team. We also thank N. Ness at Bartol Research Institute for the ACE MFI data and D. J. McComas at Southwest Research Institute for the ACE SWE data. This work was sup- ported by the Korea Science and Engineering Foundation grant R14-2002-043-01000-0. The work of K.-H. Kim was supported by the MOST grants (M1-0104-00-0059 and M1-0407-00-0001) of the Korean government.

\section{References}

Araki, T., A physical model of the geomagnetic sudden commencement, in Solar Wind Sources of Magnetospheric Ultra-Low-Frequency Waves, edited by M. J. Engebretson, K. Takahashi, and M. Scholer, pp. 183200, AGU, Washington D. C., 1994.

Balogh, A., et al., The Cluster magnetic field investigation: Overview of in-flight performance and initial results, Ann. Geophys., 19, 1207-1217, 2001.

Chen, L. and A. Hasegawa, A theory of long-period magnetic pulsations, 1. Steady state excitation of field line resonance, J. Geophys. Res., 79, 1024-1032, 1974.

Glassmeier, K.-H., M. Hönisch, and J. Untiedt, Ground-based and satellite observations of traveling magnetospheric convection twin vortices, $J$. Geophys. Res., 94, 2520-2528, 1989.

Greenwald, R. A. and A. D. M. Walker, Energetics of long period resonant hydromagnetic waves, Geophys. Res. Lett., 7, 745-748, 1980.

Gustafsson, G., et al., The electric field and wave experiment for the Cluster mission, Space Sci. Rev., 79, 137-156, 1997.

Harrold, B. G. and J. C. Samson, Standing ULF modes of the magnetosphere: A theory, Geophys. Res. Lett., 19, 1811-1814, 1992.

Harvey, P., et al., The electric field instrument on the Polar satellite, in The Global Geospace Mission, edited by C. T. Russell, pp. 583-596, Kluwer Acad. Publ., Dordrecht, 1995.

Hoppe, M. et al., Upstream hydromagnetic waves and their association with backstreaming ion populations: ISEE 1 and 2 observations, Monochromatic ULF wave excitation in the dipole magnetosphere, $J$. Geophys. Res., 86, 4471-4492, 1981.

Kataoka, R., H. Fukunishi, S. Fujita, T. Tanaka, and M. Itonaga, Transient response of the Earth's magnetosphere to a localized density pulse in the solar wind: Simulation of traveling convection vortices, J. Geophy. Res., 109, doi:10.1029/2003JA010287, 2004.

Kepko, L. and H. E. Spence, Observations of discrete, global magnetospheric oscillations directly driven by solar wind density variations, $J$. Geophys. Res., 108, doi:10.1029/2002JA009676, 2003.

Kepko, L., H. E. Spence, and H. J. Singer, ULF waves in the solar wind as direct drivers of magnetospheric pulsations, Geophys. Res. Lett., 29, doi:10.1029/2001GL014405, 2002.

Kim, K.-H. et al., Magnetospheric response to sudden and quasiperiodic solar wind variations, J. Geophys. Res., 107, doi:10.1029/2002JA009342, 2002.

Korotova, G. I. and D. G. Sibeck, Generation of ULF magnetic pulsations in response to sudden variations in solar wind dynamic pressure, in Solar Wind Sources of Magnetospheric Ultra-Low-Frequency Waves, edited by M. J. Engebretson, K. Takahashi, and M. Scholer, pp. 265271, AGU, Washington D. C., 1994.

Lee, D.-H., Dynamics of MHD wave propagation in the low-latitude magnetosphere, J. Geophys. Res., 101, 15,371-15,386, 1996.

Lee, D.-H. and R. L. Lysak, Monochromatic ULF wave excitation in the dipole magnetosphere, J. Geophys. Res., 96, 5811-5817, 1991.

Lee, D.-H. and R. L. Lysak, Numerical studies on ULF wave structures in the dipole model, in Solar Wind Sources of Magnetospheric Ultra-LowFrequency Waves, edited by M. J. Engebretson, K. Takahashi, and M. Scholer, pp. 293-297, AGU, Washington D. C., 1994.

Lühr, H. et al., Westward moving dynamic substorm features observed with the IMAGE magnetometer network and other ground-based instruments, Ann. Geophys., 16, 425-440, 1998.

Mathie, R. A. and I. R. Mann, Observations of Pc5 field line resonance azimuthal phase speeds: A diagnostic of their excitation mechanism, $J$. Geophys. Res., 105, 10,713-10,718, 2000.

Mathie, R. A., F. W. Menk, I. R. Mann, and D. Orr, Discrete field line resonances and the Alfvén continuum in the outer magnetosphere, Geophys. Res. Lett., 26, 659-662, 1999.

Motoba, T., T. Kikuchi, H. Lühr, H. Tachihara, T.-I. Kitamura, K. Hayashi, and T. Okuzawa, Global Pc5 caused by a DP 2-type ionospheric current system, J. Geophys. Res., 107, doi:10.1029/2001JA900156, 2002.

Pedersen, A., et al., Four-point high time resolution information on electron densities by the electric field experiments (EFW) on Cluster, Ann. Geophys., 19, 1483-1489, 2001.

Potemra, T. A. et al., Multisatellite and ground-based observations of transient ULF waves, J. Geophys. Res., 94, 2543-2554, 1989. 
Ruohoniemi, J. M., R. A. Greenwald, K. B. Baker, and J. C. Samson, HF radar observations of Pc5 field line resonances in the midnight/early morning MLT sector, J. Geophys. Res., 96, 15,697-15,710, 1991.

Russell, C. T. et al., The GGS/Polar magnetic fields investigation, in The Global Geospace Mission, edited by C. T. Russell, pp. 563-582, Kluwer Acad. Publ., Dordrecht, 1995.

Samson, J. C. and G. Rostoker, Latitude-dependent characteristics of highlatitude Pc4 and Pc5 micropulsations, J. Geophys. Res., 77, 6133-6144, 1972.

Samson, J. C., J. A. Jacobs, and G. Rostoker, Latitude-dependent characteristics of long-period geomagnetic micropulsations, J. Geophys. Res., 76, 3675-3683, 1971.

Samson, J. C. et al., Magnetometer and radar observations of MHD cavity modes in the Earth's magnetosphere, Can. J. Phys., 69, 929-937, 1991.

Samson, J. C. et al., Field line resonances associated with MHD waveguides in the magnetosphere, Geophys. Res. Lett., 19, 441-444, 1992a.

Samson, J. C. et al., Substorm intensifications and field line resonances in the nightside magnetosphere, J. Geophys. Res., 97, 8495-8518, 1992 b.

Scudder, J. D., X. Cao, and F. S. Mozer, Photoemission current-spacecraft voltage relation: Key to routine, quantitative low-energy plasma measurements, J. Geophys. Res., 105, 21,281-21,294, 2000.

Shue, J.-H. et al., Magnetopause location under extreme solar wind conditions, J. Geophys. Res., 103, 17,691-17,700, 1998.

Sibeck, D. G., Transient and quasi-periodic (5-15 min) events in the outer magnetosphere, in Solar Wind Sources of Magnetospheric Ultra-LowFrequency Waves, edited by M. J. Engebretson, K. Takahashi, and M. Scholer, pp. 173-182, AGU, Washington D. C., 1994.

Sibeck, D. G. et al., The magnetospheric response to 8-minute period strong-amplitude upstream pressure variations, J. Geophys. Res., 94, 2505-2519, 1989.

Singer, H. J., et al., Monitoring space weather with the GOES magnetometers), in GOES-8 and beyond, Proc. SPIE Vol. 2812, edited by E. R. Washwell, pp. 299-308, SPIE, Bellingham, 1996.

Southwood, D. J., Some features of field line resonances in the magneto- sphere, Planet. Space Sci., 22, 483-491, 1974.

Spreiter, J. R., A. L. Summers, and A. Y. Alksne, Hydromagnetic flow around the magnetosphere, Planet. Space Sci., 14, 223-253, 1966.

Stephenson, J. A. and A. D. M. Walker, HF radar observations of Pc5 ULF pulsations driven by the solar wind, Geophys. Res. Lett., 29, doi:10.1029/2001GL014291, 2002.

Takahashi, K. and B. J. Anderson, Distribution of ULF energy $(f<80$ $\mathrm{mHz}$ ) in the inner magnetosphere: A statistical analysis of AMPTE CCE magnetic field data, J. Geophys. Res., 97, 10,751-10,773, 1992.

Takahashi, K., S.-I. Ohtani, W. J. Hughes, and R. R. Anderson, CRRES observation of Pi2 pulsations: Wave mode inside and outside the plasmasphere, J. Geophys. Res., 106, 15,567-15,581, 2001.

Tsyganenko, N. A., A magnetospheric magnetic field model with a warped tail current sheet, Planet. Space Sci., 37, 5-20, 1989.

Walker, A. D. M., R. A. Greenwald, W. F. Stuart, and C. A. Green, Stare auroral radar observations of Pc5 geomagnetic pulsations, J. Geophys. Res., 84, 3373-3388, 1979.

Waters, C. L., K. Takahashi, D.-H. Lee, and B. J. Anderson, Detection of ultralow-frequency cavity modes using spacecraft data, J. Geophys. Res., 107, doi:10.1029/2001JA000224, 2002.

Yeoman, T. K., D. K. Milling, and D. Orr, Pi2 pulsation polarization patterns on the U. K. sub-auroral magnetometer network (SAMNET), Planet. Space Sci., 38, 589-602, 1990.

Yumoto, K. et al., Propagation mechanism of daytime Pc 3-4 pulsations observed at synchronous orbit and multiple ground-based stations, $J$. Geophys. Res., 90, 6439-6450, 1985.

Ziesolleck, C. W. S. and D. R. McDiarmid, Statistical survey of auroral latitude Pc 5 spectral and polarization characteristics, J. Geophys. Res., 100, 19,299-19,312, 1995.

S.-K. Sung (e-mail: sksung@astro.snu.ac.kr), K.-H. Kim, D.-H. Lee, K. Takahashi, C. A. Cattell, M. André, Y. V. Khotyaintsev, and A. Balogh 